\title{
Differences between radio-loud and radio-quiet gamma-ray pulsars as revealed by Fermi
}

\section{Y. Hui *}

Department of Astronomy \& Space Sciences, Chungnam National University, Daejeon 34134, South Korea

E-mail: cyhuiecnu.ac.kr

\section{Jongsu Lee ${ }^{\dagger \ddagger}$}

Department of Astronomy, Space Science and Geology, Chungnam National University, Daejeon 34134, South Korea

E-mail: skyljs1234@naver.com

\section{J. Takata ${ }^{8}$}

Institute of Particle physics and Astronomy, Huazhong University of Science and Technology

\section{W. $\mathbf{~ N g}$}

Department of Physics, University of Hong Kong, Pokfulam Road, Hong Kong

\section{K. S. Cheng 9}

Department of Physics, University of Hong Kong, Pokfulam Road, Hong Kong

\begin{abstract}
By comparing the properties of non-recycled radio-loud (RL) $\gamma$-ray pulsars and radio-quiet (RQ) $\gamma$-ray pulsars, we have identified the differences between these two populations. We found that the $\gamma$-ray spectral curvatures of RQ pulsars are significantly larger than those of RL pulsars. The distributions of the magnetic field strength at the light cylinder $B_{\mathrm{LC}}$ are also found to be different in these two populations. We have also confirmed the previously reported difference of $\gamma$-ray to X-ray flux ratios, though the significance can be hampered by their statistical uncertainties. While positive correlation between $B_{\mathrm{LC}}$ and the cutoff energies has been found in the RQ pulsars, such relation is absence in the RL population. In the context of outer gap model, we discuss the expected properties of these two populations and compare with the differences identified in our analysis.
\end{abstract}

7th Fermi Symposium 2017

15-20 October 2017

Garmisch-Partenkirchen, Germany

\footnotetext{
${ }^{*}$ CYH is supported by the National Research Foundation of Korea grant 2016R1A5A1013277.

${ }^{\dagger}$ Speaker.

$\ddagger \mathrm{JL}$ is supported by BK21 plus Chungnam National University.

$\S$ JT is supported by the NSFC grants of China under 11573010.

๑ KSC are supported by a 2014 GRF grant of Hong Kong Government under HKU 17300814P.
} 


\section{Introduction}

Thanks to the launch of the Fermi Gamma-Ray Space Telescope, our understanding of $\gamma$-ray pulsars has taken a big leap in the last decade. In comparison with its predecessors, Large Area Telescope (LAT) on board Fermi has the improved sensitivity and is capble to determine the source location with much higher accuracy. Shortly after operation of LAT, 16 new $\gamma$-ray pulsars were detected through blind pulsation searches [1]. By the time of writing, LAT has already detected 205 gamma-ray pulsars. ${ }^{1}$

By now, the sizes of the $\gamma$-ray population of non-recycled RL and RQ pulsars are found to be comparable. The enlarged sample of both classes enable meaningful comparison of their physical properties. Previous work [2] has showed the $\gamma$-ray to X-ray flux ratios $F_{\gamma} / F_{X}$ of RQ pulsars are larger that that of their RL counterparts. Another investigation suggests the distributions of rotational period of these two populations can possibly be different [3].

The aforementioned studies have shown that the properties of RL and RQ $\gamma$-ray pulsars can be intrinsically different. However, a thorough comparison of other pulsar characteristics, such as magnetic field strength and spectral properties, is missing. This motivates us to perform a systematic search for the difference in the emission and physical properties of these two populations through a detailed statistical analysis. The details of our investigation can be found in Hui et al. (2017) [4]. In this short article, we would like to highlight the major results in our work.

\section{Highlights of our results}

All the data used in our analysis are collected from the $2^{\text {nd }}$ Fermi LAT pulsar catalog (2PC) [5] and the $3^{\text {rd }}$ Fermi LAT $\gamma$-ray point source catalog (3FGL) [6]. We searched for the possible differences between the RL and RQ $\gamma$-ray pulsar populations by applying two-sample AndersonDarling (AD) test to the unbinned distributions of various chosen parameters.

Among all the tested parameters, the most significant difference between the RQ and RL populations is the distributions of their Curve_Significance ( $p$-value $=2 \times 10^{-4}$, see Figure 1$)$. This parameter is obtained by comparing the spectral fits with a power-law with an exponential cutoff and a simple power-law model. The larger Curve_Significance is, the more significant for the presence of spectral cutoff. Our result shows that the $\gamma$-ray spectral shapes of RQ and RL pulsars are fundamentally different.

Another interesting result comes from the comparison of their magnetic field strength (Figure 2). While the distributions of surface field strength $B_{s}$ of RQ and RL pulsars are comparable ( $p$-value $=0.8$ ), significant difference have been found for their field strength at the light cylinder $B_{L C}$ ( $p$-value=0.002). We have also confirmed the difference of $F_{\gamma} / F_{x}$ between these two populations $\left(p\right.$-value $\left.=5 \times 10^{-4}\right)$ which has been previously reported by [2]. However, we found that the significance for this difference can drop considerably when the uncertainties of $F_{\gamma} / F_{x}$ are taken into account $(p$-value $=0.03)$.

We have also carried out a non-parametric correlation analysis and found that the cutoff energy $E_{\text {cut }}$ and $B_{L C}$ of RQ pulsars are strongly correlated (Spearman rank coefficient $\rho=0.7, p$ -

\footnotetext{
${ }^{1}$ For updated statistics, please refer to https://confluence.slac.stanford.edu/display/GLAMCOG/Public+List+of+LATDetected+Gamma-Ray+Pulsars
} 


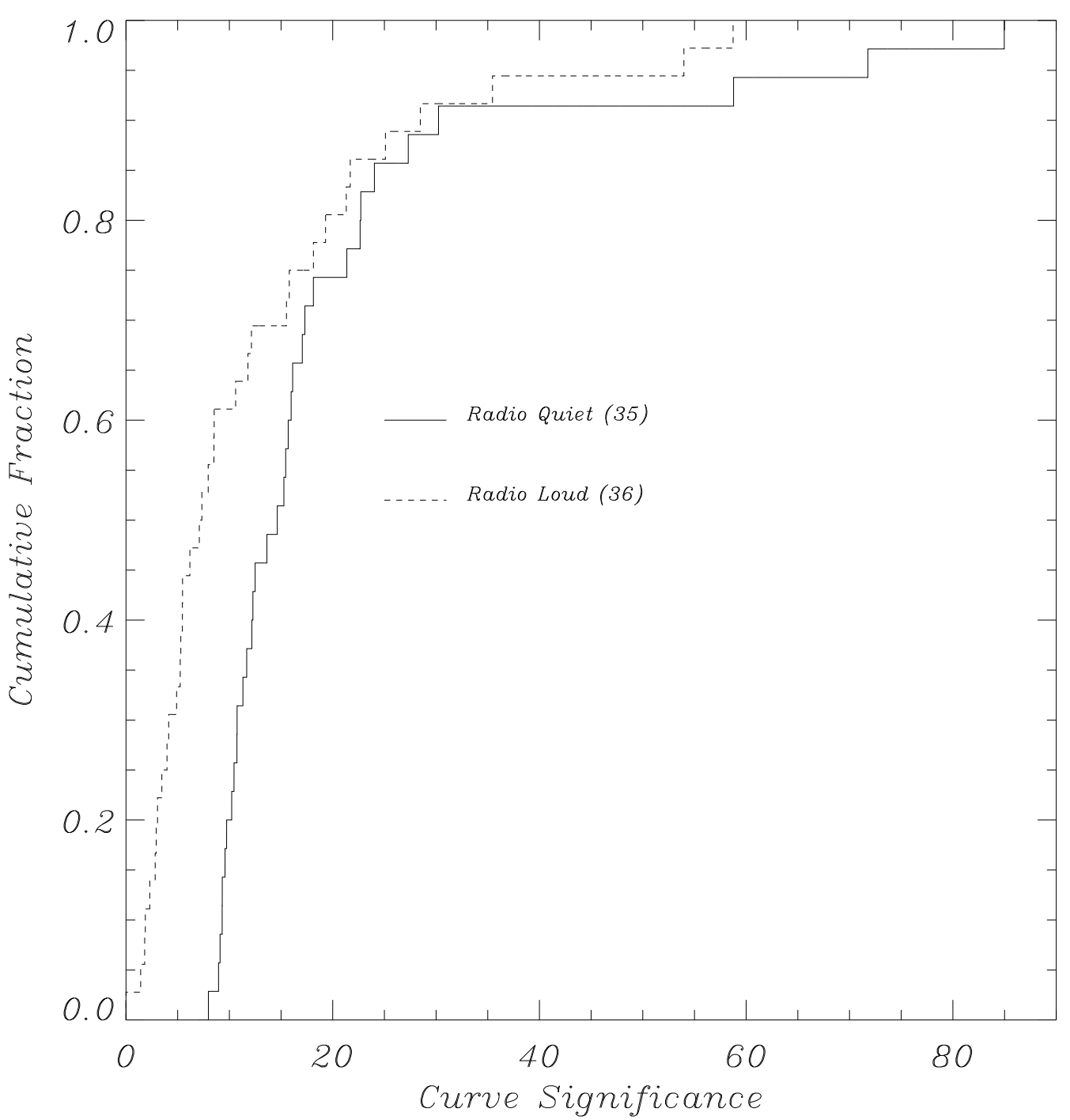

Figure 1: Cumulative distribution functions of the parameter Curve_Significance for RQ and RL $\gamma$-ray pulsars. The sample sizes of each population are given in the plot.

value $\left.=2 \times 10^{-6}\right)$. On the other hand, there is no evidence for such correlation can be found in the RL population $(\rho=0.3, p$-value $=0.1)$. Our results are consistent with [5]. We further performed a regression analysis and yield a best-fit relation of:

$$
E_{\text {cut }}=(1.15 \pm 0.11) \log B_{\mathrm{LC}}-(1.74 \pm 0.36) \mathrm{GeV}
$$

for the RQ population.

\section{Discussion}

All the aforementioned differences between RL and RQ population can be explained with 
the following assumptions: (i) the $\gamma$-rays are originated from the outer gap, (ii) the $\mathrm{X}$-rays are originated from the polar gap, and (iii) the open angle of the radio emission cone varies with the rotational period $P$ as $\sim P^{1 / 2}$.

Since $B_{\mathrm{LC}} \sim B_{\mathrm{S}} P^{-3}$, the difference of $B_{\mathrm{LC}}$ between RL and RQ populations should stem from $P$. We found that $P$ of RL population is generally shorter than that of RQ pulsars ( $p$-value $=0.006$ ). Assuming all pulsars have radio cones, whether a pulsar is RL or RQ depends on whether the line of sight can meet the radio cone. From our assumption (iii), pulsars with smaller $P$ should have wider radio cones. Hence, RQ pulsars generally have narrower radio cone than that of RL pulsars which might explain the radio-quietness of this population.

By assumption (ii), the X-ray intensity from polar cap, $F_{X}^{P C}$ depends on the angle $\theta$ between the magnetic axis and the viewing angle (i.e. $F_{X}^{P C} \propto \cos \theta$ ). Since the line of sight of RQ pulsars should be outside the radio cone, they should have a larger $\theta$ than their RL counterparts. This implies that $F_{x}$ of RL populations is larger than $F_{X}$ of RQ populations. Given that there is no significant difference of $F_{\gamma}$ between RL and RQ pulsars [7], $F_{\gamma} / F_{x}$ of RQ population should be larger.
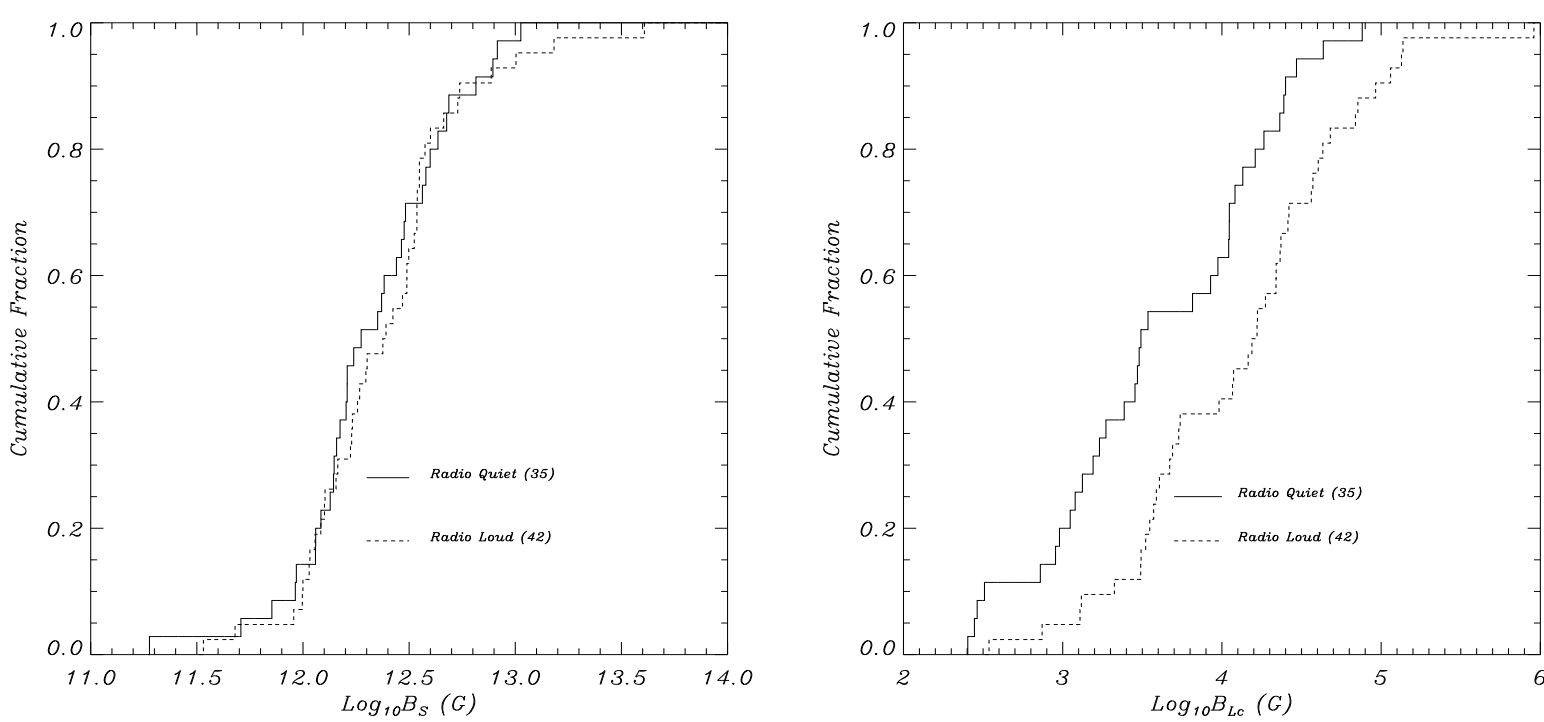

Figure 2: Cumulative distribution functions of the magnetic field strength for RQ and RL $\gamma$-ray pulsars at their stellar surface (left panel) and at their light cylinders (right panel).

To account for the difference of $\gamma$-ray spectral curvature between RL and RQ populations, we speculate that the inverse Compton (IC) process between the primary $e^{-} / e^{+}$from the outer gap and the radio photons may play a role in producing the observed high energy $\gamma$-rays. Given the narrow radio cones of RQ pulsar, the chance for their radio photons to enter the gap is low which might result in a shortage of photons produced at higher energies through IC scattering. This might explain why the $\gamma$-ray spectra of RQ pulsars are more curved than their RL counterparts.

The aforementioned IC process can also play a role in determining $E_{\text {cut }}$. This effect can be enhanced when the open angle of the radio cone is larger. Therefore, $E_{\text {cut }}$ should be proportional to $1 / P$ and hence leads to a positive correlation between $E_{\text {cut }}$ and $B_{\mathrm{LC}}$. Such correlation has been 
found in the RQ population but is absence in the RL population. This might indicate that the factors of determining $E_{\text {cut }}$ is more complex in the case of RL pulsars.

\section{References}

[1] A. A. Abdo et al. Detection of 16 Gamma-ray Pulsars Through Blind Frequency Searches Using the Fermi LAT Science, 325, 840 (2009) [arXiv:1009.0748]

[2] M. Marelli, et al. Radio-quiet and Radio-loud Pulsars: Similar in Gamma-Rays but Different in $X$-Rays ApJ, 802, $78(2015)$

[3] E.V. Sokolova, \& G.I. Rubtsov, Search for differences between radio-loud and radio-quiet gamma-ray pulsar populations with Fermi-LAT data ApJ, 833, 271 (2016) [arXiv:1601.00330]

[4] C.Y. Hui, et al. Differences between Radio-loud and Radio-quiet Gamma-ray Pulsars as Revealed by Fermi ApJ, 834, 120 (2017) [arXiv:1611.07428]

[5] A.A. Abdo, et al.The Second Fermi Large Area Telescope Catalog of Gamma-ray pulsars ApJS, 208, 17 (2013) [arXiv:1305.4385]

[6] F. Acero, et al. Fermi Large Area Telescope Third Source Catalog ApJS, 218, 23 (2015) [arXiv:1501.02003]

[7] J. Takata, Y. Wang, \& K.S. Cheng, Population Study for $\gamma$-ray Pulsars with the Outer Gap Model ApJ, 726, 44 (2011) [arXiv: 1010.5870] 